TITLE:

\title{
Multidimensional scaling and inverse distance weighting transform for image processing of hydrogeological structure in rock mass
}

\section{$\operatorname{AUTHOR}(S)$ :}

Mito, Yoshitada; Ismail, Mohd Ashraf Mohamad; Yamamoto, Takuji

\section{CITATION:}

Mito, Yoshitada ...[et al]. Multidimensional scaling and inverse distance weighting transform for image processing of hydrogeological structure in rock mass. Journal of Hydrology 2011, 411(1-2): 25-36

\section{ISSUE DATE:}

2011-12

URL:

http://hdl.handle.net/2433/151715

\section{RIGHT:}

(C) 2011 Elsevier B.V.; This is not the published version. Please cite only the published version.; この論文は出版社版でありません。引用の際に は出版社版をご確認ご利用ください。 


\section{Multidimensional Scaling and Inverse Distance Weighting Transform for Image Processing \\ 2 \\ of Hydrogeological Structure in Rock Mass}

3

4

5

6
Yoshitada Mito ${ }^{\mathrm{a} *}$, Mohd Ashraf Mohamad Ismail ${ }^{\mathrm{b}}$, Takuji Yamamoto $^{\mathrm{c}}$,

${ }^{a}$ Department of Urban Management, Graduate School of Engineering, Kyoto University, Nishikyo-ku, Kyoto, 6158530, Japan.

mito@kumst.kyoto-u.ac.jp

Tel: +8175-383-3319; Fax: +8175-383-3318

${ }^{b}$ School of Civil Engineering, Universiti Sains Malaysia, Engineering Campus, 14300, Nibong Tebal, Seberang Prai Selatan, Pulau Pinang, Malaysia.

mashrafbmismail@yahoo.com

${ }^{c}$ Kajima Corporation, 19-1, Tobitakyu 2-Chome, Chofu-shi, Tokyo 182-0036, Tokyo, 1820036, Japan.

yamataku@kajima.com

\section{Abstract}

A new imaging method based on the multidimensional scaling (MDS) and inverse distance weighting (IDW) transform is proposed in this study. This method aims to identify, characterize and process an image of the preferential flow path in a rock mass, which strongly governs the hydraulic behavior of this rock mass. This methodology uses pair-wise hydraulic diffusivity data from cross-hole hydraulic testing as the input data. The input data are then processed by MDS and IDW to generate a spatial distribution map of the hydraulic properties, which can be used to 
24 infer the preferential flow path in the rock mass. The reliability of this novel method was

25 validated through numerical experiments using several continuum models with different

26 hydrogeological structures, and the applicability of the developed method to the actual field was

27 verified through in-situ experiments.

28 Keywords: Preferential flow path; Multidimensional scaling; Inverse distance Weighting; Cross-

29 hole hydraulic test

\section{Introduction}

31 The performance of underground facilities such as high-level waste repositories and

32 underground storage caverns depends on the hydraulic behavior of the surrounding rock mass

33 (Chung et al., 2003; Giramonti et al., 1978; Lindblom, 1989; Runchal and Maini, 1980; Tiren et

34 al., 1999). The performance evaluation for such kinds of underground facilities normally focuses

35 on the potential fluid transport from or to the surrounding hydrogeological environment, where

36 the fluid flows are primarily governed by the hydraulic properties of the surrounding rock and

37 the preferential flow paths embedded in the rock mass (Bonin et al., 2000; Kiyoyama, 1990;

38 Kjorholt and Broch, 1992; Zimmerman and Bodvarsson, 1996).

39 In a rock mass, fluid flow is often concentrated or localized in certain flow paths, which

40 heavily influence hydraulic behavior (Park et al., 2002; Wang and Kulatilake, 2008). Identifying

41 these flow paths is therefore essential. However, knowledge of the presence and the connectivity

42 of the flow paths, especially the super conductive fracture, is generally quite difficult to obtain

43 due to the heterogeneity of the rock mass.

44 To grasp the heterogeneity of the hydraulic properties of rock masses, various hydraulic tests

45 based on point-wise measurements have been developed, including the Lugeon test, pumping test, 
46 slug test and constant head injection test (Black, 1978; Bouwer and R.C., 1976; Butler and Zhan,

47 2004; Houlsby, 1976; Kipp, 1985; Pickens et al., 1987). These tests deliver point-wise data that

48 require a spatial interpolation to estimate the properties at unsampled sites in the area

49 surrounding the sampled points (Cassiani et al., 1998). However, the hydraulic behavior of a

50 rock mass can vary by several orders of magnitude within the short distances between fractures

51 and the matrix, thus increasing the uncertainty of the interpolation.

52 In general practice, the most conductive fractures at the borehole scale are identified based on

53 the core logs and borehole television (BTV) observations. Subsequently, intensive packer testing

54 is performed in multiple boreholes, and pressure variations in multiple intervals in the

55 observation boreholes are monitored by the cross-hole hydraulic test (Martinez-Landa and

56 Carrera, 2006). The sequential step is needed because the single borehole only provides

57 information about the properties of the fracture segments surrounding the borehole, whereas the

58 cross-hole hydraulic test provides information on the properties of the flow zones that connect

59 borehole pairs. The pair-wise hydraulic measurements obtained by the cross-hole hydraulic test

60 enable the evaluation of the hydraulic connectivity in rock masses (Le Borgne et al., 2007). In

61 this context, hydraulic diffusivity is regarded as the key indicator of connectivity between two or

62 more boreholes. Zones with a larger connectivity and diffusivity can act as a preferential flow

63 path and have a substantial impact on the subsurface fluid flow and transport (Becker and

64 Guiltinan, 2010; Black et al., 1986; Black and Kipp, 1981; Hsieh, 1987; Hsieh and Neuman,

65 1985; Hsieh et al., 1985).

66 In the past few years, the interpretation of such hydraulic features has been largely improved

67 through advanced hydraulic tests, such as hydraulic tomography (Liu et al., 2002; Yeh and Liu,

68 2000; Zhu and Yeh, 2005). However, this method is time consuming and computationally 
69 intensive because of the inversion required to estimate the spatial distribution of hydraulic

70 properties between the tested holes (Meier et al., 2001). In most cases, the inversion results are

71 strongly influenced by the initial model, the accuracy of which is difficult to assume, especially

72 in three-dimensional cases. In addition, the flow path, which is actually an assembly of several

73 segments or vector-based objects, is often represented as an assembly of grid cells, causing

74 unavoidable errors due to the size and geometry of the grid cells.

75 In this study, MDS and the IDW transform are implemented for the image processing of

76 hydrogeological structures. These images are derived from the pair-wise hydraulic measurement

77 data set from the cross-hole hydraulic tests. MDS is a set of related statistical techniques and is

78 used here to gain insight in the relations between the pair-wise hydraulic measurement data, as

79 MDS provides a geometrical representation of these relations (Kruskal and Wish, 1978).

80 Meanwhile, IDW, a commonly used interpolation technique, is used to perform a spatial

81 interpolation of the point-wise data generated with MDS (Shepard, 1964; Wackernagel, 1998).

82 The MDS and IDW transform is then used to identify, characterize and process an image of

83 the preferential flow path in the rock mass. The reliability and applicability of the method were

84 validated through a series of numerical experiments and verified through in-situ experiments.

\section{Methodology}

87 The pair-wise hydraulic diffusivity data from cross-hole hydraulic tests are used as the input

88 data. These input data are then processed by MDS and IDW to obtain an image of the

89 preferential flow path. The following sub-sections provide details on the data acquisition and

90 data processing procedures. 
91 2.1. Data acquisition by cross-hole hydraulic testing

92 The cross-hole hydraulic test is a method to assess the hydraulic behavior between two or

93 more boreholes (Hsieh, 1987; Hsieh et al., 1985; Martinez-Landa and Carrera, 2006). Each

94 borehole is subdivided into several intervals using packers (multi-packer system). During the test,

95 fluid is injected into an interval and the hydraulic head response is recorded at other monitoring

96 intervals.

97 The injection and monitoring intervals are regarded as the points during data processing. The

98 hydraulic diffusivities (hydraulic conductivity divided by specific storage) between an injection

99 interval and monitoring intervals are determined from the hydraulic head at the injection interval,

100 the temporal change in hydraulic heads at the monitoring intervals, and the distance between the

101 injection interval and the monitoring intervals.

102 The cross-hole hydraulic test is often carried out using an injection borehole as an injection

103 interval and the remaining boreholes as monitoring intervals. This configuration of data points is

104 applied to geo-tomography in the geophysical field. However, this configuration is not essential

105 for cross-hole hydraulic testing. Monitoring intervals can be set in the injection borehole as well

106 because indirect fluid pressure propagation along the angular flow path is rather common,

107 whereas elastic waves propagate in an almost straight line.

108 To perform a detailed analysis, it is desirable to obtain a matrix of hydraulic diffusivities

109 between all pairs of test intervals by setting monitoring intervals in both the monitoring and the

110 injection boreholes in the cross-hole hydraulic test. A matrix of the hydraulic diffusivities

111 between all test intervals, which were based on the scheme of the cross-hole hydraulic test shown

112 in Fig. 1, is illustrated in Table 1. The fluid injection pressure in the cross-hole hydraulic test is 
113 normally specified as a constant by the constant pressure injection test or sinusoidal by

114 sinusoidal pressure test.

\section{Fig. 1}

\section{2.1.1. Constant pressure injection test}

118 In the constant pressure injection test, the injection and monitoring intervals are treated as a

119 pair of points. The head response in the monitoring intervals as a result of the constant pressure

120 injection in the injection intervals can be obtained through graphical interpretation of the test

121 results by conventional curve matching (Hsieh, 1987; Hsieh and Neuman, 1985; Hsieh et al., 122 1985).

123 Labeling the distance between the injection point and the monitoring point as $R$, the solution

124 for the injection point and monitoring point can be written as (Hsieh and Neuman, 1985):

125

$$
\Delta h=\frac{Q\left[K_{d}\right]^{1 / 2}}{4 \pi R D^{1 / 2}} \operatorname{erfc}\left[\left(\frac{R^{2} S_{s}}{4 K_{d} t}\right)^{1 / 2}\right\rceil
$$

126

$$
D=\left|\begin{array}{ll}
K_{11} & K_{12} \\
K_{21} & K_{22}
\end{array}\right|,
$$

127 where $\Delta h$ is the head increase at the monitoring point, $Q$ is the volumetric injection rate at the 128 injection point, $t$ is time elapsed, $S_{s}$ is the specific storage of the rock mass, $D$ is the determinant 
129 of $K$ as expressed in (2) and $K_{d}$ is the directional hydraulic conductivity between the injection

130 and monitoring intervals.

131 The dimensionless form of (1) is expressed as:

132

$$
\Delta h_{P D}=\operatorname{erfc}\left[1 / 4\left(t_{D}\right)^{1 / 2}\right]
$$

133 where $\Delta h_{P D}$ is the change in the dimensionless hydraulic head and $t_{D}$ is the dimensionless time,

134 which are, respectively, defined as:

135

$$
\Delta h_{P D}=(4 \pi R \Delta h / Q)\left[D / K_{d}\right]^{1 / 2}
$$

136 and

137

$$
t_{D}=K_{d} t /\left(R^{2} S_{s}\right)
$$

138 To analyze the constant pressure injection test results, a theoretical type curve of $\Delta h_{P D}$ versus

$139 t_{D}$ was prepared on a log-log plot according to (4) and (5).

140 Then the following steps are performed for each monitoring interval $j$ :

$141 \quad$ i. Plot $\Delta h_{j}$ versus $t$ on a log-log plot with log cycles of the same size as the type curve.

142 ii. Superimpose the data on the type curve by keeping the coordinate axes of the two plots parallel to obtain the best fit between the in-situ test data and the theoretical type curve (Fig. 2). 
145 iii. Choose an arbitrary match point anywhere on the overlapping portion of the two log-log 146 graphs and denote the corresponding values of $\Delta h_{j}, \Delta h_{P D}, t$ and $t_{D}$ by $\Delta h_{j}^{*}, \Delta h_{P D}{ }^{*}, t^{*}$ and $t_{D}^{*}$ (Fig. 2).

148 Substitute the above quantities into (4) and (5) to compute:

149

$D / K_{d}=\left(Q_{j} \Delta h_{P D}^{*} / 4 \pi R_{j} \Delta h_{j}^{*}\right)^{2}$,

150 where $D$ is the determinant of $K_{d}$, and the directional diffusivity is expressed by:

151

$K_{d} / S_{s}=R_{j} t_{D}^{*} / t^{*}$

\section{Fig. 2}

153 By matching the measured head response to the type curve on the log-log plot, the directional

154 hydraulic diffusivity $K_{d} / S_{s}$ can be computed from the constant pressure injection test. An 155 example of the hydraulic diffusivity configuration computed from the constant pressure injection 156 test is shown in Table 1.

$157 \quad$ 2.1.2. Sinusoidal pressure test

158 The sinusoidal pressure test is a cross-hole technique in which a small zone of one borehole is 159 subjected to a sinusoidal variation of pressure while a similar zone in an adjacent borehole is 160 monitored (Black and Kipp, 1981). The pressure variation in the source zone is created by 
161 careful injection and abstraction. This sinusoidally varying pressure is detected in the receiver

162 zone. The amplitude of the pressure variation is smaller in the receiver zone than in the source

163 zone because the pressure waves require some time to diffuse from the source to the receiver.

164 The decrease in amplitude and the phase lag of the received signal compared to the source signal

165 depend on the geometry and hydrogeological properties of the flow paths, which can indirectly

166 be employed to compute the hydraulic diffusivity of the rock mass (Barker, 1988; Black et al.,

167 1986; Black and Kipp, 1981; Motojima et al., 1993).

168 The ratio of the amplitude attenuation $\left|G^{*}\right| /\left|G_{0}^{*}\right|$ of the sinusoidal pressure and the time lag of

169 the phase (phase lag) $\Phi^{*}$ can be obtained by the following equations (Black and Kipp, 1981):

170 Amplitude attenuation,

171

$$
\frac{\left|G^{*}\right|}{\left|G_{0}^{*}\right|}=\frac{N_{0}\left[r\left(\frac{\omega}{\kappa}\right)^{1 / 2} \mid\right.}{N_{0}\left[r_{0}\left(\frac{\omega}{\kappa}\right)^{1 / 2}\right]} \text {, and }
$$

172 Phase lag,

173

$$
\Phi^{*}=\Phi_{0}\left[r\left(\frac{\omega}{\kappa}\right)^{1 / 2}\right\rceil,
$$

174 where $N_{0}$ is the amplitude of the Kelvin function, $r$ is the distance from the point or line source,

$175 r_{0}$ is the radius of sphere or cylinder enveloping the source, $\omega$ is the frequency of the periodic

176 functions and $\kappa$ is the hydraulic diffusivity. 
177 The hydraulic diffusivity $\kappa$ can be obtained by measuring the amplitude attenuation $\left|G^{*}\right| /\left|G_{0}{ }^{*}\right|$

178 or the phase shift $\Phi^{*}$. The sinusoidal pressure test thus allows estimation of the hydraulic

179 diffusivity without measuring the flow rate during a testing period. Moreover, the resulting

180 diffusivity values are not influenced by either the initial groundwater pressure or any changes.

181 This test can be used for estimating the three-dimensional hydraulic continuity of joints and

182 cracks. To this end, the pressure-receiving boreholes must be placed in the three-dimensional

183 region around the source borehole (Motojima et al., 1993). The equipment required for

184 performing the sinusoidal pressure tests is well described by Holmes, 1984; Holmes and

185 Sehlstedt, 1985 and Motojima et al., 1993.

2.2. Hydraulic configuration of the test intervals

The configuration of the test intervals in a cross-hole hydraulic test can be expressed by

188 Cartesian coordinates, which represent the distances between the intervals in the real

189 geographical space. In our study, the configuration of test intervals is also expressed in an

190 alternative coordinate system: in this case, the coordinates do not represent geographical

191 distances but hydraulic distances, which are proportional to the travel time of water over this

192 distance. These hydraulic coordinates can be considered inside an imaginary hydraulic subspace,

193 where the hydraulic distance is measured.

194 The reciprocal of hydraulic diffusivity is a variable appropriate to represent the hydraulic 195 distance, just as "slowness" is the reciprocal of velocity in the tomography case. The hydraulic 196 distance $D_{h}$ is defined as the time elapsed for the hydraulic diffusion $K / S_{s}$ to occur per unit 197 distance $l$ (length) and can be expressed by: 
198

$$
D_{h}=\left(\frac{K / S_{s}}{l}\right)^{-1} \text {. }
$$

199 A matrix of the hydraulic distance, as shown in Table 2, can be obtained from the matrix of 200 the hydraulic diffusivities between the pairs of test intervals shown in Table 1.

\section{Table 2}

\subsubsection{Multidimensional scaling}

MDS was used in this study to express the configuration of the hydraulic distance between test intervals (Table 2) as coordinates in an imaginary hydraulic subspace.

205 MDS is a mathematical procedure by which information contained in a data set can be

206 represented by points in a space. Essentially, the purpose of the MDS is to provide a visual 207 representation of the pattern of proximities (i.e., similarities or distances) among a set of objects.

208 MDS plots objects on a map such that objects that are very similar to each other are placed

209 near each other on the map and objects that are very different from each other are placed far 210 away from each other on the map (Kruskal and Wish, 1978; Naugpal, 2001).

211 The main output of MDS is a spatial representation of a geometric configuration of points on

212 a map. Each point in the configuration corresponds to one object. The configuration on the map

213 can reveal the hidden structure of the data and make the data much easier to comprehend.

214 MDS can also be used indirectly to analyze data that are not real proximities but that can 215 nevertheless be expressed as proximities. The hydraulic distance that was computed from the 
216 hydraulic diffusivities obtained from the cross-hole hydraulic test can therefore be considered an

217 expression of proximity.

218 Several algorithms to determine a configuration of a set of objects have been suggested in

219 MDS. Kruskal's algorithm (Kruskal and Wish, 1978), which is widely applied, is adopted in this

220 study.

221 In MDS, each object is represented by a point, where $x_{i}$ is used to indicate the point that

222 corresponds to the $i^{\text {th }}$ object. $X$ is used to indicate the entire configuration of points from

$223 x_{i} \ldots \ldots \ldots x_{l}$. In this kind of configuration, the distance between the points of $X$ plays a central

224 role. The distance between two points $x_{i}$ and $x_{j}$ is indicated by:

225

$d_{i j}=d\left(x_{i}, x_{j}\right)$

226 where $d_{i j}$ is the distance from $x_{i}$ to $x_{j}$.

227 Not all proximities between all pairs of data $d_{i j}$ must be known to obtain the hydraulic

228 configuration, because MDS is a statistical method that is able to estimate missing proximity

229 values. This feature is of practical significance because actual field data sets often suffer from

230 missing observations.

231 With the MDS method, a configuration of data points in the hydraulic subspace can be 232 obtained from the matrix of hydraulic distances (Table 2), as shown in Fig. 3. Although it is quite

233 difficult to imagine the fundamental structure of the flow path from the matrix of hydraulic

234 diffusivities in Table 1 and the hydraulic distances in Table 2, the graphical expression from the 
235 MDS in Fig. 3 gives insight into all hydraulic relationships between and among the points (in the

236 cross-hole hydraulic test) and the approximated hydro-geological structure. For example, the

237 existence of principal pathways between A5 and B3 can be inferred at a glance in Fig. 3.

\section{Fig. 3}

2.3. Geographical configuration of equally spaced obstacle points in the hydraulic subspace

240 In the hydraulic subspace, the hydraulic diffusivity is constant. This way, "obstacles" for fluid

241 flow (like clay particles in soil), which are represented in the hydraulic subspace by a set of

242 equally spaced points, can easily be detected. What needs to be done now is to relocate these

243 obstacles in the original geographical space, so that we can locate the obstacles in the real world.

244 Therefore, we need to refer to the relationship between the geographical and hydraulic

245 configurations of data points, which is assumed as follows (see Fig. 4).

246 i. The transformation between two spaces is implemented in an imaginary geometric hyperspace, where the dimensionless geometric distance is measured. The relative configuration of points in this imaginary geometric hyperspace is identical with the relative configurations of the corresponding points in the geographical and in the

$250 \quad$ hydraulic space.

251 ii. The area (volume) of the target polygon (polyhedron), which is surrounded by data points, is the same in the three considered spaces (geographical, hydraulic and imaginary geometric).

254 iii. The configuration of the target polygons in the geometric hyperspace is determined such that the barycenters of the target polygons (polyhedrons) are identical in the three spaces 
256 (geographical, hydraulic, and imaginary geometric) and such that the three 257 representations of a given data point are as aligned as possible.

258 With these assumptions, the configuration of a set of equally spaced points in the 259 geographical space can be determined by spatial interpolation using IDW in the geometric 260 hyperspace.

\section{Fig. 4}

\subsubsection{Inverse distance weighting}

263 The IDW method is the simplest and most widely used spatial interpolator based on the 264 spatial correlation between scattered points (Shepard, 1964; Wackernagel, 1998). IDW estimates 265 the value at any unsampled site by weighting the available data samples by a power of $p$, the 266 inverse distance between the sampled location and the unsampled location (scaling the weights

267 to be a unit sum). In the inverse distance weighted interpolation, the interpolation value of $Z\left(x^{\prime}\right)$

268 in the position $x$ is calculated based on the following expression:

269

$$
Z\left(x^{\prime}\right)=\sum_{i=1}^{n} w_{i} Z\left(x_{i}\right)
$$

270 where $n$ is the number of scatter points (data samples) in the set, $Z\left(x_{i}\right)$ are the values at the

271 sampled points (e.g., the data values set), and $w_{i}$ are the weights assigned to each sampled point.

272 This weight is calculated with the following function: 
273

$$
w_{i}=\frac{h_{i}^{-p}}{\sum_{j=1}^{n} h_{j}^{-p}},
$$

274 where $p$ is a positive real number called the power parameter (typically, $p=2$ ) and $h_{i}$ is the

275 distance from the sampled location to the unsampled location for which an interpolated value is 276 sought.

277 The weight function varies from a value of unity at the sampled site to a value approaching 278 zero at an infinite distance of the sampled location, so that samples lying closer to the unsampled 279 site receive a higher weight. The weight function is normalized such that the weights sum to 280 unity. The vector from a point in the hydraulic space to the corresponding point in the 281 geographical space is given by a linear combination of the vectors linking the locations of the 282 sampled sites in the hydraulic space to their corresponding geographical locations. A 283 configuration of a set of equally spaced points in the geographical space can be obtained from 284 the configurations, as shown in Figure 5.

\section{Fig. 5}

287 The spatial density of obstacle points in the geographical space is considered to represent the 288 degree of impermeability of the rock. Therefore, regions with a lower density of obstacle points 289 correspond to preferential flow paths. 
290 From the results of the preliminary parametric study (see Section 3), the relationship between

291 the density of obstacle points and hydraulic diffusivity was clarified. This relationship enables

292 the estimation of the hydraulic diffusivity at an arbitrary location within the target polygon in the

293 geographical space. If the specific storage capacity of the rock is known, the spatial distribution

294 of the hydraulic conductivity can be found.

295 Several kinds of methods can be considered to measure and display the spatial density of the

296 points. For instance, it can be assessed as the number of obstacles per cell of a superimposed

297 raster. Another approach consists of counting the number of obstacles within a circular

298 neighborhood, which results in a continuous spatial density distribution.

\section{Fig. 6}

300 2.5. Three-dimensional model

301 The proposed two-dimensional methodology can easily be applied to the three-dimensional

302 context, which involves the three-dimensional geographical space, hydraulic subspace and

303 geometric hyperspace. In the three-dimensional cases, the target polygon in the two-dimensional

304 cases is generalized as the target polyhedron.

305 Because both MDS and IDW are intrinsically multidimensional methods, the three-

306 dimensional space continua in the form of a polyhedron can be transformed in a similar way as

307 the two-dimensional one. The hydraulic properties can be evaluated from the volumetric spatial

308 density of obstacle points in the geographical space. Fig. 7 shows the scheme of the three309 dimensional method. 


\section{Fig. 7}

\section{3. Verification of the methodology}

\section{3.1. Numerical experiments}

313 A series of numerical experiments was conducted to verify the applicability of the proposed 314 method and investigate its performance.

315 Two-dimensional and three-dimensional continuum models, simulating different positions of 316 a super-conductive flow path with a certain width, were made in a region with a horizontal width

317 of $110 \mathrm{~m}$ and a height of $80 \mathrm{~m}$, as shown in Fig. 8. A hydraulic conductivity of $10^{-5} \mathrm{~m} / \mathrm{s}$ was 318 given to the super-conductive flow path, whereas $10^{-7} \mathrm{~m} / \mathrm{s}$ was given to the surrounding rocks. A 319 uniform specific storage of $10^{-3} \mathrm{~m}^{-1}$ was assumed throughout the model region.

320 The sides and the top of the region were head-specified boundaries. At the bottom of the 321 region, an impermeable boundary was assumed. The numerical cross-hole test with a constant 322 injection pressure was conducted using seven test intervals with 10-m holes.

323 The hydraulic diffusivity between each pair of test intervals was determined from the 324 computed temporal changes in the hydraulic head, which were obtained from the finite element 325 analysis of the unsteady state groundwater flow.

326 The hydraulic configuration of test intervals was determined by MDS using hydraulic

327 distances between all pairs of test intervals. Sequentially, the configuration of obstacles was 328 determined by IDW, and then the image of the flow path was obtained by counting the number 329 of obstacles per cell of the raster, as shown in Fig. 8. 


\section{Fig. 8}

331 Fig. 9 shows several sets of the original hydrogeological structure, the hydraulic configuration

332 of data points and the computed flow path image in a case of a horizontal flow path intersecting

333 the target polygon at several depths. The pixels with a lower density (higher hydraulic

334 conductivity) were drawn with a lighter gray in the computed image.

335 The horizontal line-shaped flow path emerged at the appropriate position. In addition, the 336 hydraulic configuration gave not only the hydraulic relationship between (and/or among) data 337 points but also an integrated interpretation of the test results in light of the actual phenomena.

\section{Fig. 9}

339 Figs. 10 and 11 show cases where an oblique conductive path intersects the target polygon.

340 Although Fig. 9 shows that the method can be applied successfully for horizontal flow paths,

341 there are some differences in performance in the cases of oblique and vertical conductive paths

342 (Fig. 10 and 11). The preferential flow path in the second example in Fig. 11 is less accurately

343 reproduced compared to the other examples: the zone visualized as conductive flow path not

344 only contains the path but also includes a substantial part of its surroundings. Meanwhile, the

345 vertical flow paths shown in Fig. 12 cannot be visualized at all. Nevertheless, the computed

346 pattern of hydraulic configuration data points and equally spaced obstacle points shown in Fig.

34712 (b) hints at the presence of a preferential flow path.

Fig. 10

349

Fig. 11 
351 Fig. 13 shows how the presence of multiple flow paths is visualized. This is important for the

352 evaluation of the hydraulic behavior of a real fracture system because such a system often

353 contains multiple flow paths. As for the horizontal and the oblique fracture patterns, appropriate

354 images were also obtained in this case. The image of the multiple flow paths can be used not

355 only to assess the physical connections but also to gain insight into the hydraulic connections

356 between individual fractures, which influence the fluid flow.

\section{Fig. 13}

358 Three-dimensional models are important for constructing a fracture network model that is 359 accurate to the real structures. Fig. 14 shows several original hydrogeological structures, the

360 hydraulic configurations of data points corresponding with these structures and the computed

361 flow path images. The original hydrogeological structures represent cases where a horizontal

362 flow path intersects the target polygon at different depths, similar to the case of the two-

363 dimensional models shown in Fig. 9. Fig. 15 shows the results obtained for oblique conductive

364 paths intersecting both sides of the target polygon. In the computed images, the lighter gray

365 colors indicate zones with a higher hydraulic conductivity. Because the images obtained by the

366 two-dimensional and three-dimensional numerical experiments show the assumed flow path at

367 the appropriate location, it can be assumed that the proposed imaging method is reliable. 


\section{4. Applicability of the methodology}

\section{4.1. In-situ experiments}

The applicability of the imaging method, which shows good performance in the numerical

374 three-dimensional models.

375 4.1.1. Field experiment in tertiary sedimentary rocks - two-dimensional case

376 A cross-hole hydraulic test with constant injection pressure as explained in Section 2.1.1 was

377 conducted using two boreholes, which were drilled in a tertiary alternation of mudstone, 378 sandstone and pumice tuff with a homoclinal structure.

379 The geographical configuration of the nine test intervals and the imaging results given by the 380 proposed method were drawn on the underlying geological profile, as shown in Fig. 16. The 381 objective polygon contains mudstone, pumice tuff layers and sandstone sub-layers I, II and III.

382 The image of the hydrogeological structure is composed of several layers with different

383 hydraulic properties. The direction of these layers corresponds with the underlying geological 384 structure. Furthermore, the sandstone layer II and the pumice tuff layers, where joints are denser 385 show a higher permeability compared to the other geological layers. Moreover, in zones with 386 mudstone and sandstones I and III, where the joints are more sparse, a lower permeability is 387 indicated. This means that the proposed method provides an appropriate two-dimensional image 388 of the hydrogeological structure of the rock mass in this particular case. 
390 4.1.2. Field experiment in cretaceous sedimentary rocks - three-dimensional case

391 The cross-hole hydraulic test with sinusoidal pressure, as explained in Section 2.1.2, was

392 conducted using three boreholes, which were drilled in a cretaceous alternation of sandstone and

393 mudstone. Fig. 17 (a) shows the geographical configuration of the eleven source and observation

394 intervals, which were set at a depth of $25 \mathrm{~m}$ to $35 \mathrm{~m}$ from the exploration adit of the underground

395 power station construction site. The presence of a steep fracture zone in the target polyhedron is

396 confirmed by both the adit wall observation and the borehole television (BTV) observation (Fig.

39717 (a)), although this fracture zone does not directly appear at any source or observation interval.

398 Fig. 17 (b) shows the hydraulic configuration of the data points. Fig. 17 (c) shows the computed

399 image of the preferential flow path. This image shows good agreement with the fracture zone,

400 which is the only hydrogeological structure in the target polyhedron that can be a super 401 conductive flow path. This means that the proposed methodology is able to deliver a reliable 402 three-dimensional image for this field test.

Fig. 17

\section{5. Discussion and conclusion}

405 Preferential flow paths are ubiquitous and always play an important role in the hydraulic

406 behavior of a rock mass. The influence of these flow paths on the hydraulic behavior of a rock 407 mass is difficult to characterize due to the inherent complexities in fracture network geometries, 
408 densities and connectivity. These factors often dominate the selection of the methodology used

409 for the detection, characterization and visualization of flow paths.

410 In this study, a new method for visualizing the hydrogeological structure in rock masses that

411 is simpler than computationally intensive inference or inversion was developed. The method was

412 validated by numerical and in-situ experiments. It does not require any kind of initial model and

413 is able to determine the geometric and hydraulic properties of the flow paths, such as orientation,

414 shape and hydraulic conductivity, in both two and three dimensions.

415 Although the method can be applied successfully, there are some differences in performance 416 between the various situations presented in this study. The method appears to perform well for 417 detecting the preferential flow path if there is a difference in hydraulic diffusivity between the 418 point pairs involved in the cross-hole test. From the numerical analysis results, it was found that 419 the degree of intersection determines whether the preferential path will have a large or a small 420 impact on the measured diffusivity. The higher the degree of intersection (i.e., in the case of a 421 horizontal flow path), the larger the impact on the measured hydraulic diffusivity will be. In case 422 of such a high degree of intersection, the preferential flow path can be clearly visualized. 423 However, if the degree of intersection is smaller (i.e., an oblique flow path) or similar for all 424 point pairs, as in the vertical flow path cases, the calculated diffusivity for all pairs will be 425 similar, and thus the preferential flow path cannot be visualized.

426 The possible orientation of the preferential flow path needs to be estimated before the 427 methodology developed in this study can be applied. The identification of the preferential flow 428 path orientation by means of geological mapping or geophysical techniques gives the 429 information required to determine the orientation of the boreholes for the cross-hole test, so that 
the preferential flow path can be detected. In case of a dominant vertical flow path in the study area, inclined boreholes that can intercept the vertical flow path must be included in the cross-

432 hole test.

433

434

435

\section{Acknowledgments}

The authors are most grateful to the Kajima Corporation for their assistance with the assessment of the in-situ experiments and to other persons for providing invaluable input for this paper.

\section{References}

Barker, J.A., 1988. A Generalized Radial Flow Model for Hydraulic Tests in Fractured Rock. Water Resources Research, 24(10): 1796-1804.

Becker, M.W., Guiltinan, E., 2010. Cross-hole periodic hydraulic testing of inter well connectivity, Thirty-fifth workshop on geothermal reservoir engineering, Stanford university.

Black, J.H., 1978. Use of Slug Test in Groundwater Investigations. Water Serv, 82(985): 174178.

Black, J.H., Barker, J.A., Noy, D.J., 1986. Crosshole investigations - the method theory and analysis of crosshole sinusoidal pressure tests in fissured rock, Stripa Project IR 86-03, SKB, Stockholm.

Black, J.H., Kipp, K.L., 1981. Determination of Hydrogeological Parameters Using Sinusoidal Pressure Tests - a Theoretical Appraisal. Water Resources Research, 17(3): 686-692.

Bonin, B., Colin, M., Dutfoy, A., 2000. Pressure building during the early stages of gas production in a radioactive waste repository. J Nucl Mater, 281(1): 1-14.

Bouwer, H., R.C., R., 1976. A slug test method for determining hydraulic conductivity of unconfined aquifers with completely or partially penetrating wells. Water Resources Research, 12(3): 423-428.

Butler, J.J., Zhan, X.Y., 2004. Hydraulic tests in highly permeable aquifers. Water Resources Research, 40(12): -.

Cassiani, G., Bohm, G., Vesnaver, A., Nicolich, R., 1998. A geostatistical framework for incorporating seismic tomography auxiliary data into hydraulic conductivity. J Hydrol, 206(1-2): 58-74.

Chung, I.M., Cho, W.C., Heo, J.H., 2003. Stochastic hydraulic safety factor for gas containment in underground storage caverns. J Hydrol, 284(1-4): 77-91.

Giramonti, A.J., Lessard, R.D., Blecher, W.A., Smith, E.B., 1978. Conceptual Design of Compressed Air Energy-Storage Electric-Power Systems. Appl Energ, 4(4): 231-249.

Holmes, D.C., 1984. Crosshole investigations - equipment design considerations for sinusoidal pressure tests, Stripa Project IR 84-05, SKB, Stockholm.

Holmes, D.C., Sehlstedt, M., 1985. Crosshole investigations - design of the hydraulic testing system, Symposium on in-situ experiments in granite associated with the disposal of radioactive waste. OECD Nuclear Energy Agency and SKBF, Stockholm, pp. 203-213. 
Houlsby, A.C., 1976. Routine Interpretation of the lugeon water test. Quarterly Journal of Engineering Geology and Hydro-geology, 9(4): 303-313.

Hsieh, P.A., 1987. characterizing the hydraulic properties of fractured rock massess methodology and case studies, The 28th U.S. Symposium on rock Mechanics (USRMS). A. A. Balkema, Tucsan, AZ, pp. 465-472.

Hsieh, P.A., Neuman, S.P., 1985. Field Determination of the 3-Dimensional Hydraulic Conductivity Tensor of Anisotropic Media .1. Theory. Water Resources Research, 21(11): 1655-1665.

Hsieh, P.A., Neuman, S.P., Stiles, G.K., Simpson, E.S., 1985. Field Determination of the 3Dimensional Hydraulic Conductivity Tensor of Anisotropic Media .2. Methodology and Application to Fractured Rocks. Water Resources Research, 21(11): 1667-1676.

Kipp, K.L., 1985. Type Curve Analysis of Inertial Effects in the Response of a Well to a Slug Test. Water Resources Research, 21(9): 1397-1408.

Kiyoyama, S., 1990. The present state of underground crude oil storage technology in japan. Tunneling and Underground Space Technology, 5(4): 343-349.

Kjorholt, H., Broch, E., 1992. The Water Curtain - a Successful Means of Preventing Gas Leakage from High-Pressure, Unlined Rock Caverns. Tunn Undergr Sp Tech, 7(2): 127-132.

Kruskal, J.B., Wish, M., 1978. Multidimensional scaling. Quantitative applications in the social sciences. SAGE Publication, London.

Le Borgne, T. et al., 2007. Comparison of alternative methodologies for identifying and characterizing preferential flow paths in heterogeneous aquifers. J Hydrol, 345(3-4): 134-148.

Lindblom, U., 1989. The Performance of Water Curtains Surrounding Rock Caverns Used for Gas-Storage. International Journal of Rock Mechanics and Mining Sciences \& Geomechanics Abstracts, 26(1): 85-97.

Liu, S.Y., Yeh, T.C.J., Gardiner, R., 2002. Effectiveness of hydraulic tomography: Sandbox experiments. Water Resources Research, 38(4): -.

Martinez-Landa, L., Carrera, J., 2006. A methodology to interpret cross-hole tests in a granite block. J Hydrol, 325(1-4): 222-240.

Meier, P.M., Medina, A., Carrera, J., 2001. Geostatistical inversion of cross-hole pumping tests for identifying preferential flow channels within a shear zone. Ground Water, 39(1): 10-17.

Motojima, I., Kono, I., Nishigaki, M., 1993. Crosshole permeability testing method in bedrock. Soils and Foundation, 33(4): 108-120.

Naugpal, P.S., 2001. Guide to advanced data analysis: using IDAMS software. UNESCO, Division of information and informatics.

Park, B.Y. et al., 2002. Determination of the hydraulic conductivity components using a threedimensional fracture network model in volcanic rock. Eng Geol, 66(1-2): 127-141.

Pickens, J.F., Grisak, G.E., Avis, J.D., Belanger, D.W., Thury, M., 1987. Analysis and Interpretation of Borehole Hydraulic Tests in Deep Boreholes - Principles, Model Development, and Applications. Water Resources Research, 23(7): 1341-1375.

Runchal, A., Maini, T., 1980. The Impact of a High-Level Nuclear Waste Repository on the Regional Groundwater-Flow. Int J Rock Mech Min, 17(5): 253-264.

Shepard, D., 1964. A two dimensional interpolation function for irregulallry data spaced, ACM Nat. Conf, pp. 517-524.

Tiren, S.A., Askling, P., Wanstedt, S., 1999. Geologic site characterization for deep nuclear waste disposal in fractured rock based on 3D data visualization. Eng Geol, 52(3-4): 319-346. 
512 Wackernagel, H., 1998. Multivariate Geostatistics: An Introduction With Applications. . 513 Springer-Verlag, Berlin.

Wang, M.Y., Kulatilake, P.H.S.W., 2008. Understanding of hydraulic properties from configurations of stochastically distributed fracture networks. Hydrol Process, 22(8): 11251135.

Yeh, T.C.J., Liu, S.Y., 2000. Hydraulic tomography: Development of a new aquifer test method. Water Resources Research, 36(8): 2095-2105.

519 Zhu, J.F., Yeh, T.C.J., 2005. Characterization of aquifer heterogeneity using transient hydraulic tomography. Water Resources Research, 41(7): -.

Zimmerman, R.W., Bodvarsson, G.S., 1996. Hydraulic conductivity of rock fractures. Transport Porous Med, 23(1): 1-30.

525 Fig. 1: Schematic of cross-hole hydraulic test with the superconductive flow path.

526 Fig. 2: Log-log plot of the measured head respond versus time with superimposed and fitted type

527 curves.

528 Fig. 3: Hydraulic configuration of data points and equally spaced obstacle points in the hydraulic

529 subspace.

530 Fig. 4: Hydraulic and geographical configurations of data points in the geometric hyperspace.

531 Fig. 5: Geographical configuration of obstacles points in the geographical space.

532 Fig. 6: Raster-base measurement and expression technique.

533 Fig. 7: Schematic for the three-dimensional case procedure.

534 Fig. 8: Two-dimensional and three-dimensional continuum models for finite element analysis of

535 unsteady state groundwater flow with an example of a conductive flow path.

536 Fig. 9: Comparisons between the original hydro-geological structure (a) and the computed

537 imaging result (c) in the cases where a horizontal conductive path intersects the target polygon at 
538 several depths. The pixels with lower density are drawn with a lighter gray in the computed

539 image. The hydraulic configuration of data points (b) is also shown.

540 Fig. 10: The same comparison as shown in Fig. 9 in cases where an oblique conductive path

541 intersects both sides of the target polygon.

542 Fig. 11: The same comparison as in Fig. 9 in the case where an oblique conductive path

543 intersects both the top and the bottom of the target polygon.

544 Fig. 12: The same comparison as in Fig. 9 in the case where a vertical conductive path intersects

545 both the top and bottom of the target polygon.

546 Fig. 13: The same comparison as in Fig. 9 in the case where two conductive paths intersect the 547 target polygon.

548 Fig. 14: Comparisons between the original hydro-geological structure in three dimensions (a)

549 and the computed imaging result (c) in the case where a horizontal conductive path intersects the

550 target polygon at several depths. The pixels with lower density are drawn with a lighter gray in

551 the computed image. The hydraulic configuration of data points (b) is also shown.

552 Fig. 15: The same comparison as in Fig. 14 in the cases where an oblique conductive path

553 intersects both sides of the target polyhedron in the three-dimensional cases

554 Fig. 16: Layout of the test intervals and the imaging result on a geological map for the two-

555 dimensional case. The sandstone layer that is located in the mid of the objective polygon can be

556 subdivided into three sub-layers (I, II and III) from the point of view of rock properties. 
557 Fig. 17: Layout of the test interval and the image of the preferential flow path for the three-

558 dimensional case. The fracture zone as inferred in (a) is strongly associated with the image

559 obtained in (c).

560 Table captions

561 Table 1: An illustration of a matrix of hydraulic diffusivities between all the test intervals (unit:

$\left.562 * 10^{-4} \mathrm{~cm}^{2} / \mathrm{s}\right)$

563 Table 2: Matrix of the hydraulic distance, which is obtained from the matrix of hydraulic

564 diffusivities as shown in Table 1 (unit: s/cm). 